\title{
Tissue Prognostic Factors in Melanoma: Expression of B7 Family Members B7-H3 and B7-H4 and Death Receptors TRAIL-R1 and TRAIL- R2
}

\author{
Hélène Aubert-Wastiaux ${ }^{1,2}$, Anne-Chantal Knol ${ }^{2}$, Amir Khammari ${ }^{1,2}$, Mélanie Saint-Jean ${ }^{1,2}$, \\ Jean-Michel Nguyen ${ }^{3}$, Gaelle Quéreux ${ }^{1,2}$ and Brigitte Dreno ${ }^{*}, 1,2$
}

\author{
${ }^{I}$ Unité de Cancéro-Dermatologie-CIC biothérapie INSERM 0503 CHU Hôtel-Dieu, 1 place Alexis Ricordeau \\ 44093Nantes cedex 1, France \\ ${ }^{2}$ Inserm Unit U892, 9 quai Moncousu 44093 Nantes cedex 1 France \\ ${ }^{3}$ SEB, CHU Nantes Hopital Saint-Jacques, 85 rue Saint-Jacques 44093 Nantes cedex 1, France
}

\begin{abstract}
High resistance rate of melanoma to chemotherapy results in the development of other therapeutic strategies such as immunotherapy. Discrepancies observed in clinical responses to immunotherapy suggest the presence of immune polymorphisms and tumor escape mechanisms.

The objective of this study was to investigate the expression of B7-H3 and B7-H4 which are major stakeholders in immune regulation, and of the death receptors TRAIL-R1 and TRAIL-R2 which play an important role in controlling tumor cell proliferation and apoptosis. The correlation between the expression levels of these proteins and the clinical outcome has also been assessed.

The expression level of the proteins was analyzed on 32 invaded LN samples and on 11 matching tumor cell lines. Immunohistochemical staining and double fluorescent immunostaining on lymph node (LN) frozen sections were performed, as well as flow cytometry on tumor cell lines.

The 4 proteins were expressed in all LN biopsies within a range from $40.6 \%$ for TRAIL-R1 to $87.5 \%$ for TRAIL-R2. These 4 proteins were rarely expressed by the tumor cell lines except for TRAIL-R2 which was detected in 10 of the 11 cell lines. The expression of these proteins by tumor cell lines is not correlated with in vivo expression found in immunohistochemistry (IHC). It raises the question of the role of the tissue environment in the expression of the proteins. The expression levels of B7-H4, TRAIL-R1, and TRAIL-R2 using IHC were correlated with overall survival. In vivo, high expression levels of B7-H4, TRAIL-R1 or TRAIL-R2 appear as poor prognostic factors.
\end{abstract}

Keywords: Melanoma, Immunotherapy, B7, TRAIL-R1, TRAIL-R2, Prognostic marker, Lymph node metastasis.

\section{INTRODUCTION}

The treatment of loco regional metastasis in melanoma relies on surgery associated or not with adjuvant therapies like administration of interferon, while for distant metastasis, the treatment consists of chemotherapy with limited effectiveness and a response rate of less than $15 \%$ [1].

Surgery, associated or not with adjuvant therapies like administration of interferon, is used in the treatment of loco regional metastasis in melanoma. As for distant metastasis, the treatment consists of chemotherapy with limited effectiveness and a response rate of less than $15 \%[1]$. Other therapeutic strategies have then been developed in melanoma and especially adoptive (TILs) and active (vaccination) immunotherapy, based on the high immunogenic potential of melanoma [2-4]. Trials are currently underway on this kind of approach, but clinical benefits are still limited. It appears

\footnotetext{
*Address correspondence to this author at the Skin Oncology Department, CIC Biotherapy INSERM 0503, CHU Hotel Dieu, 1 place Alexis Ricordeau, 44035 Nantes cedex 1, France; Tel: 0033240083118; Fax:
} 0033210083117; E-mail: Brigitte.dreno@wanadoo.fr that response to both TILs and vaccines depends on patient subpopulations as suggested by the results of Dreno et al., [5] who showed the benefit of immunotherapy using TIL infusion among patients with only one invaded lymph node (LN). These discrepancies suggest that there might be polymorphisms of immune cells, and/or establishment of tumor escape mechanisms. Today prognostic success factors for this type of immunotherapy are only a few.

Antigen recognition by TCR on T lymphocytes underlies immune response, however optimal and complete activation of naïve $\mathrm{T}$ cells requires a secondary signal, known as costimulation. After the initial activation, co-inhibitory molecules are engaged in order to restrain $\mathrm{T}$ cell functions. Expression of co-stimulatory and co-inhibitory molecules at the appropriate times and locations positively and negatively control priming, growth, differentiation, and functional maturation of $\mathrm{T}$ cell response. B7 family members act as key positive or negative control regulators of $\mathrm{T}$ cell activation.

Using phylogenic analysis, B7 family can be divided into three groups [6]. Group I includes B7-1, B7-2 and ICOS-L (B7-H2), group II consists of PD-L1 and PD-L2, and group 
III consists of B7-H3 and B7-H4, the newest members of the B7 family. Tumor cell expression of PD-L1 as a poor prognostic factor has been established for several tumors (kidney, ovary, gastric, breast, bladder, and pancreas)[7-12]. More recently the correlation between the level of PD-L1 expression and progression of the disease in melanoma has been reported [13].

B7-H4 seems to have an inhibitory function [14] but its receptor remains unknown. B7-H3 can promote or inhibit Tcell response. Only the activating receptor is known. The expression of $\mathrm{B} 7-\mathrm{H} 3$ and $\mathrm{B} 7-\mathrm{H} 4$ are reported on different tumor cell types [15]. B7-H3 and B7-H4 expression have been reported on melanoma cells in vivo and in vitro [16]. Their prognostic role has been established in other tumors such as colorectal carcinoma, renal cell carcinoma or prostate cancer [17-19]. More recently a survival benefit for patient with low B7-H4 expression in melanoma was found whereas B7-H3 was not associated with clinical parameter in this study [16].

The death receptors tumor necrosis factor-related apoptosis inducing ligand receptors 1 and 2 (TRAIL-R1 and TRAIL-R2) also play an important role in controlling tumor cell proliferation and in tumor escape as they can induce apoptosis of the tumor cell. The level of apoptosis induced by TRAIL appears to be largely determined by the level of expression of the death receptors [20] but the latter's expression on melanoma tumor cells, as their putative role, remains debated ( is still being debated or remains unproved), [21-23].

The objective of this study was to investigate the mechanisms of tumor escape. First we studied the expression by melanoma cells of B7-H3 and B7-H4 which may have an immunosupressive action in the immune response against melanoma, and secondly we studied the expression of TRAIL-R1 and TRAIL-R2 which modifications could be a way of resistance to apoptose. We studied those proteins expression frequency in vivo on melanoma invaded lymph nodes (LNs), and in vitro on melanoma cell lines obtained from the same LNs in order to determine if the expression of these proteins could have a prognostic value.

\section{MATERIAL AND METHOD}

\section{Material}

Metastatic melanoma tumour-invaded LNs from which TIL were obtained, were used [5, 24, 25]. After lymphadenectomy, the majority of the invaded LN samples was used to produce TIL but a biopsy fragment was collected for immunochemistry, and for the establishment of the autologous tumor cell line. The rest of the samples was processed for pathologic examination. All the patients signed a consent form approved by the Ethic committee (Nantes University Hospital).

From each of the 32 collected LN fragments, we obtained a frozen section for in situ immunohistochemistry (IHC), and autologous tumor cell lines when possible.

\section{Immunohistochemistry}

In order to obtain frozen sections, part of the lymph node fragment was embedded in TissueTek ${ }^{\circledR}$ (Sakura, Tokyo,
Japan), and $5 \mu \mathrm{m}$ thick sections were cut using a Leica cryostat. IHC was performed using the streptavidin/peroxydase technique on deep-frozen sections obtained from metastatic lymph nodes. Cryostat sections ( 5 $\mu \mathrm{m})$ were rehydrated in a Tris-buffered saline (TBS)/ bovine serum albumin (BSA) $0.1 \%$ solution (Sigma, St Quentin Fallavier, France), and endogenous peroxydase activity was blocked with an endogenous peroxidase blocking solution (DAKO, Trappes, France). The expression of the B7 family members and death receptors TRAIL-R1 and TRAIL-R2 at the protein level were detected using the following mouse monoclonal antibodies: anti B7-H3 (LS-B2614, LifeSpanBioSciences), anti B7-H4 (H74, eBiosciences), TRAIL-R1 (DJR1, eBiosciences), and anti TRAIL-R2 (DJR2-4, eBiosciences). Antibodies were used either at the concentration of $5 \mu \mathrm{g} / \mathrm{ml}$ or $10 \mu \mathrm{g} / \mathrm{ml}$ and were incubated overnight at $+4{ }^{\circ} \mathrm{C}$ and washed with $\mathrm{TBS} / 0.1 \%$ BSA 30 minutes. Primary antibody was revealed by the biotinstreptavidin/peroxydase technique (DAKO ChemTek detection kit rabbit/mouse, Trappes, France) using AEC as substrate. Briefly, biotinylated secondary antibody and streptavidin/peroxydase were successively incubated for 30 minutes at room temperature. Sections were then incubated with AEC for 5 minutes and finally counterstained with Mayer's hemalun and mounted with aqueous mounting medium. Between the different steps, tissue sections were washed in TBS $/ 0.1 \%$ BSA. Mouse monoclonal IgG1 isotype control (DAKO) was substituted to primary antibody as negative control.

Slides were read using a Leica microscope. The analysis of the sections was done by one investigator. The expression of the molecule in the tumor area was assessed in an open manner qualitatively: expression groups were defined based on presence or absence of membrane staining; and quantitatively using a semi-quantitative scale from 0 to 4 for each studied LN biopsy (0: no stained cell; 1 : 1 to $25 \%$ stained cells, 2: 25 to $50 \%$ stained cells, $3: 50$ to $75 \%$ stained cells, 4: more than 75\% stained cells). Double fluorescent immunostaining

This technique was performed for two LN biopsies in order to see exactly which types of cells were expressing the protein of interest detected by immunochemistry. Cryostat sections $(10 \mu \mathrm{m})$ were incubated overnight with the same primary antibodies than those used for immunohistochemistry. Slides were washed three times and incubated with a secondary fluorescent Alexa 488 conjugated antibody (IgG goat anti mouse, Invitrogen, CergyPontoise, France). Sections were then incubated for 2 hours with the second primary antibody Melan-A (EP1422Y, Abcam, San Francisco, USA) specific of melanoma cells, washed and incubated with another secondary fluorescent Alexa 568 conjugated antibody (IgG goat anti rabbit, Invitrogen, CergyPontoise, France). Nuclei were stained using Hoëchst(Sigma Aldrich, Saint-Louis, MO, USA). The sections were mounted with a glycerol medium: Mowiol(Polysciences, Warrington, USA). For each sample, one negative control slide was included.

Images were captured with a confocal Laser Scanning Microscope in collaboration with the Cellular and Tissular Imaging Core Facility of Nantes University (MicroPICell). 


\section{Production of Melanoma Cell Lines}

The autologous tumor cell lines were established from the metastatic LN fragment in the Nantes Unit for Cellular and Gene therapy as previously described [26]. 1 to $2 \mathrm{~mm}^{3}$ lymph node fragments were centrifuged and then pieces were inoculated on a culture medium enriched with fetal calf serum. The plates were observed under a light microscope every week and sub-cultured if necessary. The cell lines were obtained after 2 to 6 months for 40 to $70 \%$ of the LN samples, depending on the culture media used, and then frozen. The presence of melanoma cells was confirmed by analyzing the expression of tumor-associated antigens using flow cytometry and RT-PCR.

\section{Flow Cytometry Analysis}

Tumor cell lines were subjected to flow cytometry analysis. Cells were stained with phycoerythrin(PE) conjugated antibodies. The following mouse monoclonal antibodies were used: anti B7-H3 (LS-C74208, LifeSpanBioSciences), anti B7-H4 (H74, eBiosciences), antiTRAIL-R1 (DJR1, eBiosciences), anti TRAIL-R2 (DJR2-4, eBiosciences). They were used at a dilution of $1 / 40$. After half hour incubation at $+4^{\circ} \mathrm{C}$ in the presence of monoclonal antibodies or isotype control, cells were washed twice, resuspended in PBS and analyzed on a FACScan(Becton Dickinson, Mountain View, CA, USA) and using CellQuestPro software (Becton Dickinson). The mean fluorescence intensity was calculated on a log scale. A $\mathrm{PE}$ conjugated anti-Mouse $\mathrm{IgG} 1 \kappa$ was used as a negative control.

\section{Statistical Analysis}

The Fischer's exact test and Wilcoxon rank test were used to analyze the correlation between the proteins expression in IHC and in flow cytometry. A value of $\mathrm{P}<0.05$ was considered as significant.

Survival analyzes were performed. The Kaplan-Meier estimator was used. Likelihood ratio test (LRT) or Log rank test were used for each variable studied.

8 tests were used, 2 for each parameters (B7-H4, B7-H3, TRAIL-R1, TRAIL-R2). A Benjamini-Hochberg procedure was used to adjust the number of tests.

We studied relapse free survival ( the proportion of patient without known recurrence of the disease) and overall survival ( proportion of patient who stay alive. It takes into account death due to any cause).

\section{RESULTS}

Thirty-two melanoma invaded LN biopsies and 11 tumor cell lines obtained from these LNs were analyzed.

\section{Expression Levels}

\section{Immunohistochemical Expression on Melanoma Invaded LNS}

An expression of B7-H3 was found in $81.2 \%$ of the 32 melanoma invaded LNs, and B7-H4 in 50\% of the 32 melanoma invaded LNs. Concerning the death receptors, the expression of TRAIL-R1 was found in $40.6 \%$ of the 32 melanoma invaded LNs, and TRAIL-R2 was found in $87.5 \%$ of the 32 melanoma invaded LNs. The expression levels for each protein in IHC are summarized in Table 1. The staining on frozen sections is illustrated on Fig. (1, red staining).

Table 1. Immunochemistry Results

\begin{tabular}{|c|c|c|c|c|}
\hline & B7-H3 & B7-H4 & TRAIL-R1 & TRAIL-R2 \\
\hline \hline $0: 0 \%$ & 6 & 16 & 17 & 4 \\
\hline $1: 1$ to $25 \%$ & 3 & 11 & 9 & 14 \\
\hline 2: 25 to $50 \%$ & 7 & 4 & 5 & 7 \\
\hline 3: 50 to $75 \%$ & 12 & 1 & 1 & 6 \\
\hline 4: 75 to $100 \%$ & 4 & 0 & 0 & $87.5 \%$ \\
\hline$\%$ of positive LN & $81.2 \%$ & $50 \%$ & $40.6 \%$ & 1 \\
\hline
\end{tabular}

Results obtained by immunohistochemistry: number of Lymph Node (LN) samples according to the percentages of stained cells on the frozen section (using a semiquantitative scale from 0 to 4) for the 32 melanoma invaded LNs studied in IHC and for each studied protein, and total percentage of positive $\mathrm{LN}$ for each protein.

\section{Double Immunofluorescence Analysis}

A double immunofluorescent staining was performed to confirm the specific expression of proteins by melanoma cells. We highlighted an expression of 3 out of 4 studied proteins by Melan-A positive tumor cells: B7-H3, TRAILR1, and TRAIL-R2 in 2 LN samples. Fig. (2) illustrates a tumor cell that co-expresses TRAIL-R2 and Melan-A.

\section{Flow Cytometry Analysis on Tumor Cell Lines}

Eleven tumor cell lines were studied. All the tumor cell lines analyzed were obtained from the invaded LN samples studied in IHC. TRAIL-R2 expression was identified in 10/11 melanoma tumor cell lines evaluated and B7-H4, TRAIL-R1 in only $1 / 11$ of the tumor cell lines. None of the tumor cell line analyzed expressed B7-H3 in flow cytometric analysis (positive control expressed B7-H3). The results with the average percentage of stained cells in flow cytometry and the correlation with the results obtained with the other techniques performed are summarized in Table 2 . The expression of the 4 proteins by tumor cell lines was not correlated with the expression of the proteins in IHC. Fig. (3) shows representative positive results for 3 of the 11 melanoma tumor cell lines.

\section{Correlation with Clinical Data}

\section{Correlation Between Expression Levels and Survival}

The expression level of B7-H4 in vivo (IHC) correlated with overall survival ( $\mathrm{p}$-adjusted $=0.0496$, Likelihood ratio test). The expression levels of TRAIL-R1, and TRAIL-R2 in vivo (IHC) correlated with overall survival, (respectively $\mathrm{p}$ adjusted $=0.007$ and $p$-adjusted $=0.0432$, Log rank test), and the expression levels of TRAIL-R1 correlated with relapsefree survival ( $\mathrm{p}$-adjusted $=0.029$, Log rank test). Then for TRAIL-R1 and TRAIL-R2 a higher expression level using the semi-quantitative scale in $\operatorname{IHC}(2,3,4)$ was associated with a shorter survival than for lower expression levels $(0,1)$. Fig. (4) represents the Kaplan-Meier curves obtained for TRAIL-R1 and TRAIL-R2 (Log Rank tests). The expression level of B7-H3 was not correlated with survival. In vivo high expression levels of B7-H4, TRAIL-R1 and TRAIL-R2 in invaded LNs are correlated with shorter overall survival. 


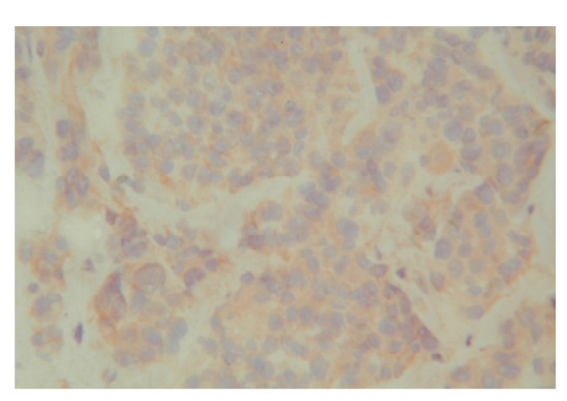

a

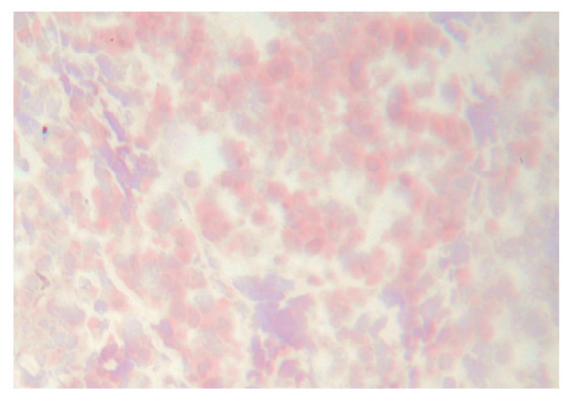

C

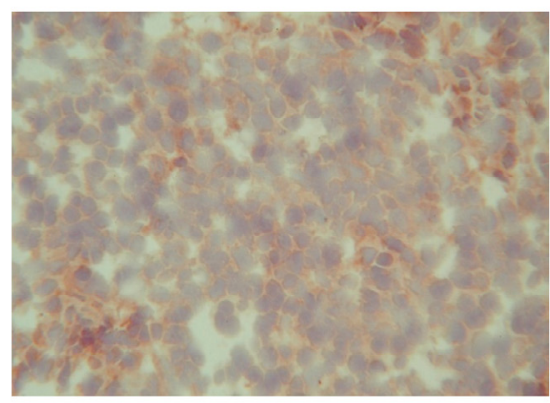

b

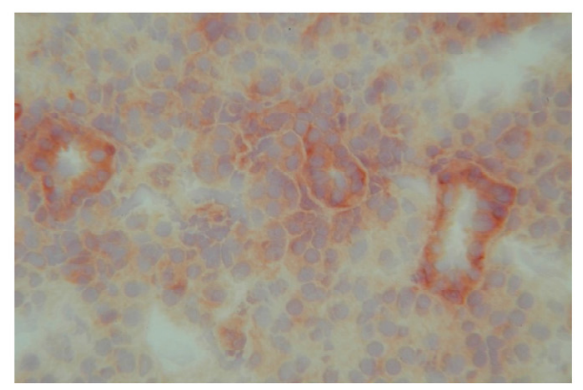

d

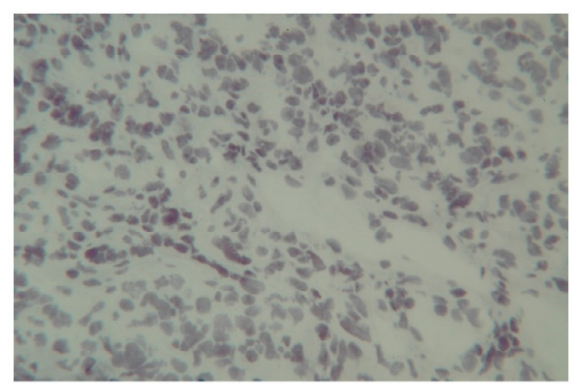

e

Fig. (1). expression levels ( 1 to 4 ) of the 4studied markers in IHC with the streptavidin/peroxydase technique (red stain) in a tumoral section of invaded lymph nodea: B7-H3 expression (x25): 1 b: B7-H4 expression (x25): 2 c: TRAIL-R1 expression (x25): 1 d: TRAIL-R2 expression (x25): 1.e: IgGisotype.

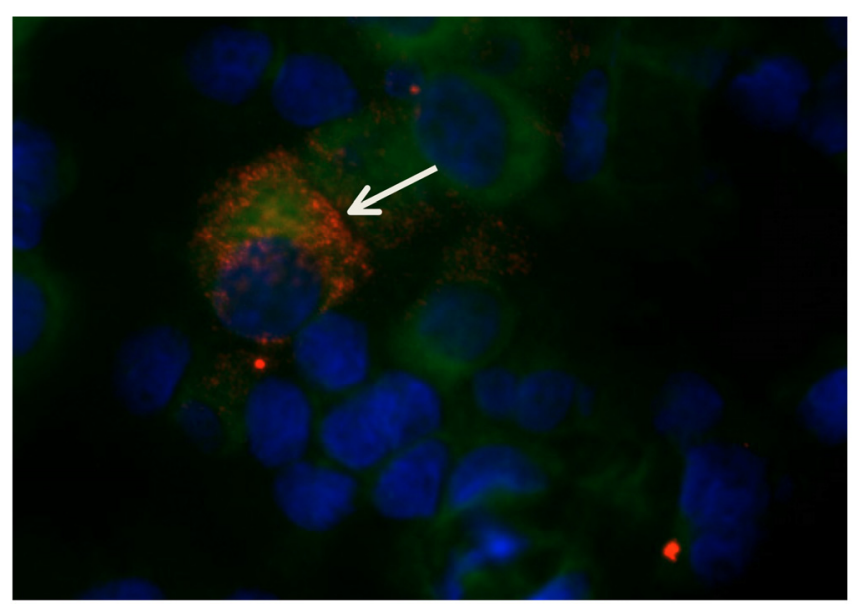

Fig. (2). co-localization of melanoma specific tumor antigen Melan-A (Texas Red) and TRAIL-R2 (FITC, green) expression in the same tumor cell (nuclei are stained with DAPI and appear in blue).

The p-values adjusted for 8 tests using the BenjaminiHochberg procedure are presented in Table 3.

\section{DISCUSSION}

In this study, we demonstrate an expression of two B7 family members B7-H3, B7-H4, and TRAIL-R1/R2by melanoma invaded LN with variable expression levels. The results of flow cytometry on tumor cell lines confirm the expression by melanoma cells of all the proteins except B7H3.
Concerning B7-H3, we found a frequent expression of this protein in invaded LN (26 out of the 32 studied LNs) associated with an absence of expression by tumour cell lines which suggest that the expression of $\mathrm{B} 7-\mathrm{H} 3$ by melanoma cells is induced by interactions with the microenvironment. The double labeling confirms this hypothesis showing that melanoma cells express B7-H3. The expression of B7-H3 by other tumor cells than melanoma has already been described $[18,27]$ and this study confirms the expression of B7-H3 by melanoma tumor cell as shown by recent data [16].

Concerning B7-H4, we found an expression by both melanoma invaded LN and melanoma cell lines. However we were unable to confirm these results by the double labeling which could either relate to a technical problem or to the fact that the in vivo expression of $\mathrm{B} 7-\mathrm{H} 4$ is related to other cells than melanoma present in the micro environment. The expression of B7-H4 by tumor cell in other cancers was already described $[14,28]$. Expression by melanoma tumor cell has also been observed [16].

Regarding the cell death receptors, we demonstrate a high percentage of expression of both TRAIL-R1 and 1 TRAIL-R2 by both invaded LN (13/24) and melanoma cell lines. In addition the expression of TRAIL R1/R2 by melanoma cells is confirmed by confocal microscopy. This expression of TRAIL R1/2 has only been reported in one previous paper (15). Therefore our work confirms the expression of the three proteins B7-H3, B7-H4, TRAIL-R1, and TRAIL-R2 by melanoma tumor cells. 
Table 2. Results Obtained with Flow Cytometry Compared to the Results Obtained with the Other Techniques

\begin{tabular}{|c|c|c|c|c|}
\hline Patient & B7-H3 & B7-H4 & TRAIL-R1 & TRAIL-R2 \\
\hline \hline 1 IHC & 3 & 1 & 1 & 3 \\
\hline DFI & + & - & + & + \\
\hline cytometry & 0 & 0 & $66,55 \%$ & $86,37 \%$ \\
\hline 2 IHC & 2 & 0 & 2 & 1 \\
\hline cytometry & 0 & 0 & 0 & $80,05 \%$ \\
\hline 3 IHC & 3 & 4 & 3 & 1 \\
\hline cytometry & 0 & 0 & 0 & $93,56 \%$ \\
\hline 4 IHC & 4 & 0 & 0 & 3 \\
\hline cytometry & 0 & 0 & 0 & $86,17 \%$ \\
\hline 5 IHC & 3 & 1 & 0 & 0 \\
\hline cytometry & 0 & 0 & 0 & $74,66 \%$ \\
\hline 6 IHC & 3 & 0 & 0 & 1 \\
\hline cytometry & 0 & 0 & 0 & 0 \\
\hline 7 IHC & 3 & 1 & 0 & 0 \\
\hline cytometry & 0 & 0 & 0 & $76,16 \%$ \\
\hline 8 IHC & 0 & 0 & 0 & 2 \\
\hline cytometry & 0 & 0 & 0 & $81,27 \%$ \\
\hline 9 IHC & 2 & 0 & 0 & 0 \\
\hline cytometry & 0 & $21,45 \%$ & 0 & $25,50 \%$ \\
\hline 10 IHC & 3 & 1 & 0 & 2 \\
\hline cytometry & 0 & 0 & 0 & $52,06 \%$ \\
\hline 11 IHC & 3 & 1 & 2 & 3 \\
\hline cytometry & 0 & 0 & 0 & $80,82 \%$ \\
\hline Average & & & & $73.66 \%$ \\
\hline
\end{tabular}

Results obtained with flow cytometry compared to the results obtained with the other techniques: expression (percentage of stained cells in flow cytometry) of the 4 studied proteins by the 11 melanoma tumor cell lines compared to the expression of the corresponding frozen sections in IHC (using a semi-quantitative scale 0 to 4), and double fluorescent immunostaining (DFI) when performed (there was no corresponding tumor cell line of the second sample used for DFI).

In addition of these results, our study demonstrates the absence of any correlation between the expression of these 2 families of proteins by tumor cell lines and invaded LN. This result supports the role of interactions between microenvironment and melanoma cells for the expression of these 4 proteins as already suggested by Nguyen et al., [22] in a previous work.

But the main originality of our work is to show that the protein expressions of three proteins among the four studied have a prognostic value in vivo in invaded lymph nodes.

A high B7-H4 expression level in invaded lymph nodes was correlated with a shorter overall survival. The prognostic value of B7-H4 has already been described in two other cancers: prostate cancer [19], and renal cell carcinoma [17] but never in melanoma. These results support the hypothesis of an inhibitory function of this protein. (a)

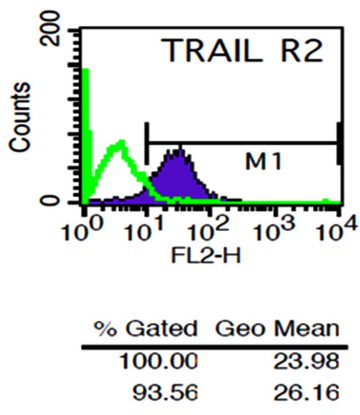

(b)

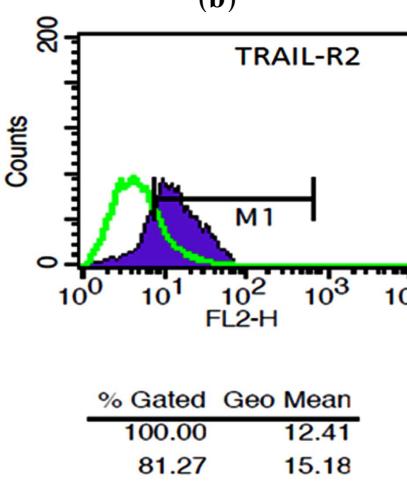

(c)

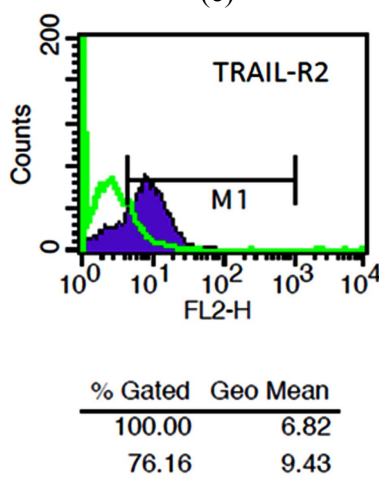

Fig. (3). a: flow cytometric analysis for the M 284 tumor cell line; b: flow cytometric analysis for the M 288 tumor cell line; c: flow cytometric analysis for the M 350 tumor cell line.

A high expression level of TRAIL-R1 or TRAIL-R2 in invaded LNs is also correlated with a shorter overall survival. The review of the results of the literature appears discordant. Indeed Zhuang et al., [20] reported that the progression of melanoma is associated with a decrease expression of both TRAIL-R1 and TRAIL-R2 [23], and McCarthy that a high TRAIL-R2 expression level correlated with increased survival [29].

However, our results are supported by studies published in other types of cancers. Indeed McCarthy and Ganten reported a higher expression rate of TRAIL-R2 associated to a shorter survival in breast cancer $[30,31]$. Cooper et al., described the same results in the non-small' cell lung cancer [32]. These discrepancies underline the complexity of interactions between TRAIL receptors and the intracellular apoptotic machinery. TRAIL receptors activation induces 
(a)

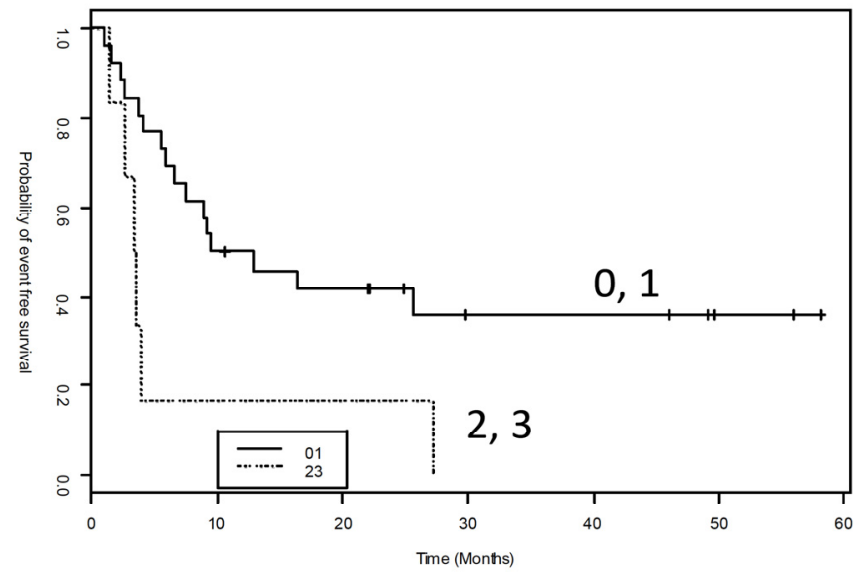

(b)

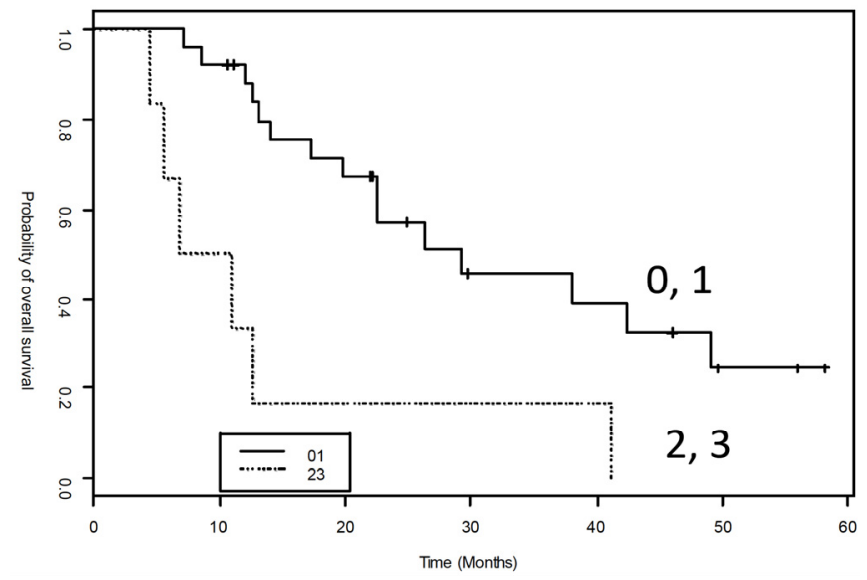

(c)

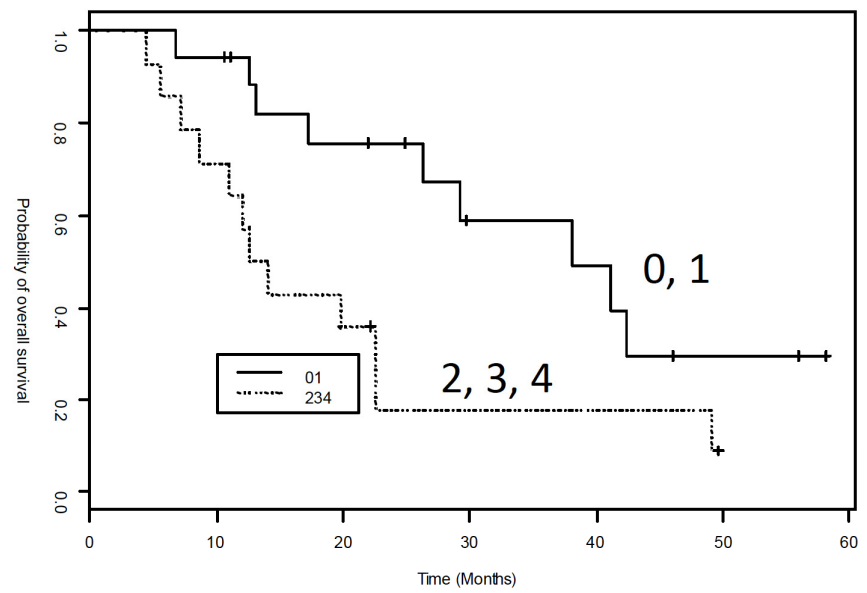

Fig. (4). Correlations between expression levels for TRAIL-R1 and TRAIL-R2 and survival (Kaplan-Meier curves). a: relapse free survival correlated with TRAIL-R1 expression level (encoded 0 and 1 vs 2, 3, 4). $\mathrm{p}=0.029$ Median of survival: group 01: 9.51 IC95\%[6.66 - NA], group 23: 3.48 IC95\% [2.66 -NA]; b: overall survival correlated with TRAIL-R1 expression level (encoded 0, 1 vs 2, 3, 4). $\mathrm{p}=0.007$ Median of survival: group 01: 29.28 IC95\% [22.49- NA], group 234: 8.95 IC95\%[5.57 -NA]; c: overall survival correlated with TRAIL-R2 expression level (encoded 0, 1 vs 2, 3, 4). $\mathrm{p}=0.0432$ Median of survival: group 01: 38.0 IC95\%[26.3 NA], group 234: 13.3 IC95\% [11-NA]. apoptosis via caspase activation. However TRAIL receptors can also activate a cell survival pathway by activating NFKB transcription factor which has an anti-apoptotic effect via the up-regulation of pro-survival genes [33]. Another hypothesis is that a part of the TRAIL-R2 expression is related to the T lymphocytes of the microenvironment with induction by tumor cells of apoptosis of these T lymphocytes in a similar manner than PDL1 expression by the melanoma cells induces an apoptosis of PDL1+ lymphocytes. Our results support the ongoing development of therapeutic agents specifically targeting TRAIL receptors or TRAIL agonists $[34,35]$.

Table 3. Benjamini-Hochberg Procedure

\begin{tabular}{|c|c|c|c|}
\hline p-Value & Order & Critical Value & Adjusted p-Value \\
\hline \hline 0.00144 & 1 & 0.00625 & 0.00724 \\
\hline 0.00181 & 2 & 0.0125 & 0.00724 \\
\hline 0.0109 & 3 & 0.01875 & 0.029067 \\
\hline 0.0216 & 4 & 0.025 & 0.0432 \\
\hline 0.031 & 5 & 0.03125 & 0.0496 \\
\hline 0.104 & 6 & 0.0375 & 0.138667 \\
\hline 0.711 & 7 & 0.04375 & 0.779 \\
\hline 0.779 & 8 & 0.05 & 0.779 \\
\hline
\end{tabular}

Benjamini-Hochberg procedure was used to adjust the number of tests (P-value: pvalue for each variable studied, Critical value: rejection threshold of the p-value, Adjusted p-value).

The main weakness of our work it that in vivo we cannot eliminate the fact that the increased expression of these 3 proteins is partially related to other cells in the tumor micro environment. But in any case, our study shows a correlation between the expression of B7-H4, TRAIL-R1 and TRAILR2 in global invaded lymph node expression (melanoma cells and micro environment) and survival of the patients making it a predictive marker of response to TILs.

In conclusion, we confirm in the present work an expression of B7-H3, B7-H4, TRAIL-R1 and TRAIL-R2 by melanoma cells. The absence of correlation between tumor tissue results and melanoma cell lines argue for the role of microenvironment in their modulation. Furthermore we show that B7-H4, TRAIL-R1 and TRAIL-R2 expression in invaded LN could be predictive marker of progression in patients treated by TILs, but these results have to be confirmed.

Our results encourage the development of therapeutics targeting the TRAIL induced apoptosis pathway.

\section{ACKNOWLEDGMENT}

Anne Laure Puaux, GSK Bio.

\section{CONFLICT OF INTEREST}

None declared.

\section{REFERENCES}

Becker JC, Kampgen E, Brocker E. Classical chemotherapy for metastatic melanoma. Clin Exp Dermatol 2000; 25(6): 503-8. 
[2] Mackensen A, Carcelain G, Viel S, et al. Direct evidence to support the immunosurveillance concept in a human regressive melanoma. J Clin Invest 1994; 93(4): 1397-402.

[3] Rosenberg SA, Spiess P, Lafreniere R. A new approach to the adoptive immunotherapy of cancer with tumor-infiltrating lymphocytes. Science 1986; 233(4770): 1318-21.

[4] Oble DA, Loewe R, Yu P, Mihm MC, Jr. Focus on TILs: prognostic significance of tumor infiltrating lymphocytes in human melanoma. Cancer Immun 2009; 9: 3 .

[5] Dreno B, Nguyen JM, Khammari A, et al. Randomized trial of adoptive transfer of melanoma tumor-infiltrating lymphocytes as adjuvant therapy for stage III melanoma. Cancer Immunol Immunother 2002; 51(10): 539-46.

[6] Seliger B, Marincola FM, Ferrone S, Abken H. The complex role of B7 molecules in tumor immunology. Trends Mol Med 2008; 14(12): 550-9.

[7] Ghebeh H, Mohammed S, Al-Omair A, et al. The B7-H1 (PD-L1) $\mathrm{T}$ lymphocyte-inhibitory molecule is expressed in breast cancer patients with infiltrating ductal carcinoma: correlation with important high-risk prognostic factors. Neoplasia 2006; 8(3): 1908.

[8] Hamanishi J, Mandai M, Iwasaki M, et al. Programmed cell death 1 ligand 1 and tumor-infiltrating $\mathrm{CD} 8+\mathrm{T}$ lymphocytes are prognostic factors of human ovarian cancer. Proc Natl Acad Sci USA $2007 ;$ 104(9): 3360-5.

[9] Inman BA, Sebo TJ, Frigola X, et al. PD-L1 (B7-H1) expression by urothelial carcinoma of the bladder and BCG-induced granulomata: associations with localized stage progression. Cancer 2007; 109(8): 1499-505.

[10] Thompson RH, Dong H, Kwon ED. Implications of B7-H1 expression in clear cell carcinoma of the kidney for prognostication and therapy. Clin Cancer Res 2007; 13(2 Pt 2): 709s-15s.

[11] Wang L, Ma Q, Chen X, Guo K, Li J, Zhang M. Clinical Significance of B7-H1 and B7-1 Expressions in Pancreatic Carcinoma. World J Surg 2010; 34(5): 1059-65.

[12] Wu C, Zhu Y, Jiang J, Zhao J, Zhang XG, Xu N. Immunohistochemical localization of programmed death-1 ligand-1 (PD-L1) in gastric carcinoma and its clinical significance. Acta Histochem 2006; 108(1): 19-24.

[13] Hino R, Kabashima K, Kato Y, et al. Tumor cell expression of programmed cell death-1 ligand 1 is a prognostic factor for malignant melanoma. Cancer 2010; 116(7): 1757-66.

[14] Zang X, Loke P, Kim J, Murphy K, Waitz R, Allison JP. B7x: a widely expressed $\mathrm{B} 7$ family member that inhibits $\mathrm{T}$ cell activation. Proc Natl Acad Sci USA 2003; 100(18): 10388-92.

[15] Sun Y, Wang Y, Zhao J, et al. B7-H3 and B7-H4 expression in non-small-cell lung cancer. Lung Cancer 2006; 53(2): 143-51.

[16] Quandt D, Fiedler E, Boettcher D, Marsch W, Seliger B. B7-H4 expression in human melanoma: its association with patients' survival and anti tumor immune response. Clin Cancer Res 2011; 17(10): 3100-11.

[17] Krambeck AE, Thompson RH, Dong H, et al. B7-H4 expression in renal cell carcinoma and tumor vasculature: associations with cancer progression and survival. Proc Natl Acad Sci USA 2006; 103(27): 10391-6.

[18] Sun J, Chen LJ, Zhang GB, et al. Clinical significance and regulation of the costimulatory molecule $\mathrm{B} 7-\mathrm{H} 3$ in human colorectal carcinoma. Cancer Immunol Immunother 2010; 59(8): 1163-71.

[19] Zang X, Thompson RH, Al-Ahmadie HA, et al. B7-H3 and B7x are highly expressed in human prostate cancer and associated with disease spread and poor outcome. Proc Natl Acad Sci USA 2007; 104(49): 19458-63.

[20] Nguyen T, Zhang XD, Hersey P. Relative resistance of fresh isolates of melanoma to tumor necrosis factor-related apoptosisinducing ligand (TRAIL)-induced apoptosis. Clin Cancer Res 2001; 7(3 Suppl): 966s-73s.

[21] Kurbanov BM, Geilen CC, Fecker LF, Orfanos CE, Eberle J. Efficient TRAIL-R1/DR4-mediated apoptosis in melanoma cells by tumor necrosis factor-related apoptosis-inducing ligand (TRAIL). J Invest Dermatol 2005; 125(5): 1010-9.

[22] Liu H, Jiang CC, Lavis CJ, et al. 2-Deoxy-D-glucose enhances TRAIL-induced apoptosis in human melanoma cells through XBP1-mediated up-regulation of TRAIL-R2. Mol Cancer 2009; 8: 122.

[23] Zhuang L, Lee CS, Scolyer RA, et al. Progression in melanoma is associated with decreased expression of death receptors for tumor necrosis factor-related apoptosis-inducing ligand. Hum Pathol 2006; 37(10): 1286-94

[24] Khammari A, Nguyen JM, Pandolfino MC, et al. Long-term follow-up of patients treated by adoptive transfer of melanoma tumor-infiltrating lymphocytes as adjuvant therapy for stage III melanoma. Cancer Immunol Immunother 2007; 56(11): 1853-60.

[25] Labarriere N, Pandolfino MC, Gervois N, et al. Therapeutic efficacy of melanoma-reactive TIL injected in stage III melanoma patients. Cancer Immunol Immunother 2002; 51(10): 532-8.

[26] Gervois N, Heuze F, Diez E, Jotereau F. Selective expansion of a specific anti-tumor CD8+ cytotoxic T lymphocyte clone in the bulk culture of tumor-infiltrating lymphocytes from a melanoma patient: cytotoxic activity and $\mathrm{T}$ cell receptor gene rearrangements. Eur $\mathrm{J}$ Immunol 1990; 20(4): 825-31.

[27] Zang X, Sullivan PS, Soslow RA, et al. Tumor associated endothelial expression of $\mathrm{B} 7-\mathrm{H} 3$ predicts survival in ovarian carcinomas. Mod Pathol 2010; 23(8): 1104-12.

[28] Yao Y, Wang X, Jin K, et al. B7-H4 is preferentially expressed in non-dividing brain tumor cells and in a subset of brain tumor stemlike cells. J Neurooncol 2008; 89(2): 121-9.

[29] McCarthy MM, DiVito KA, Sznol M, et al. Expression of tumor necrosis factor--related apoptosis-inducing ligand receptors 1 and 2 in melanoma. Clin Cancer Res 2006; 12(12): 3856-63.

[30] Ganten TM, Sykora J, Koschny R, et al. Prognostic significance of tumour necrosis factor-related apoptosis-inducing ligand (TRAIL) receptor expression in patients with breast cancer. J Mol Med 2009; 87(10): 995-1007.

[31] McCarthy MM, Sznol M, DiVito KA, Camp RL, Rimm DL, Kluger HM. Evaluating the expression and prognostic value of TRAIL-R1 and TRAIL-R2 in breast cancer. Clin Cancer Res 2005; 11(14): 5188-94.

[32] Cooper WA, Kohonen-Corish MR, Zhuang L, et al. Role and prognostic significance of tumor necrosis factor-related apoptosisinducing ligand death receptor DR5 in nonsmall-cell lung cancer and precursor lesions. Cancer 2008; 113(1): 135-42.

[33] Kimberley FC, Screaton GR. Following a TRAIL: update on a ligand and its five receptors. Cell Res 2004; 14(5): 359-72.

[34] Camidge DR, Herbst RS, Gordon MS, et al. A phase I safety and pharmacokinetic study of the death receptor 5 agonistic antibody PRO95780 in patients with advanced malignancies. Clin Cancer Res 2010; 16(4): 1256-63.

[35] Motoki K, Mori E, Matsumoto A, et al. Enhanced apoptosis and tumor regression induced by a direct agonist antibody to tumor necrosis factor-related apoptosis-inducing ligand receptor 2. Clin Cancer Res 2005; 11(8): 3126-35. 